\title{
Pace and patterns of magnetic swimmers in a billiard pool
}

\author{
Florian J. Maier, Markus Sesselmann, Ingo Rehberg, and Reinhard Richter* \\ Experimentalphysik 5, University of Bayreuth, 95440 Bayreuth, Germany
}

(Received 8 March 2017; published 6 July 2017)

\begin{abstract}
We experimentally investigate magnetic surface swimmers on water. These objects self-assemble from ferromagnetic microparticles and a nonmagnetic disk. They are floating on the liquid surface due to interface tension and move under the influence of a harmonically oscillating homogeneous magnetic field oriented vertically, which is distinguished by its amplitude and frequency. The speed of the surface swimmers strongly depends on these parameters. The functional dependencies between speed and amplitude and between speed and frequency are investigated by independently varying both control parameters. In the first case, the data obtained are in good agreement with the predicted scaling while there are some deviations in the latter case. Moreover, due to the interplay between the surface bound swimmers and the ascending liquid meniscus at the edge of the experimental vessel, different dynamics can be realized. We observe periodic and quasiperiodic trajectories in a circular vessel and aperiodic trajectories in a vessel shaped like a Bunimovich stadium.
\end{abstract}

DOI: 10.1103/PhysRevE.96.012205

\section{INTRODUCTION}

The hydrodynamics of swimming microorganisms $[1,2]$ is of great practical interest, not only for understanding their locomotion and collective behavior $[3,4]$ in various environments but also for emerging new applications at the macro, micro, and nanoscales. These include drug delivery and diagnostics [5], surgery [6], transportation [7], and mixing [8].

Among the many concepts of artificial swimmers, those utilizing time-dependent magnetic fields are particularly handy: Due to the lack of magnetic monopoles these fields are not shielded and can easily be applied over large volumes. According to the scallop theorem [9], the swimmers must execute nonreciprocal motions for low Reynolds number in a Newtonian fluid [10]. For "magnetic sperm," a chain of superparamagnetic particles linked by DNA molecules attached to a red blood cell [11], the symmetry is broken by applying a static and a time-dependent field in different directions. Swimmers with helicity, like magnetic cork-screw particles [12], inherently break the symmetry and are driven by a rotating magnetic field. Floating ferrofluidic droplets subjected to a field rotating around a horizontal axis act like paddle-steamers [13]. Here the symmetry is broken by the liquid surface. Similarly, a floating asymmetric paramagnetic doublet subjected to a precessing field [14] exploits the neighborhood of a confining wall.

In contrast to the magnetic swimmers listed above, "magnetic snakes" [16] like in Fig. 1(a) self-assemble and undergo a spontaneous symmetry breaking: Uniformly distributed micron-sized nickel particles, which are supported by the liquid-air interface, are subjected to an alternating magnetic field, oriented vertically. Due to a complex interplay of the dipoles, the field, and the surface waves, the particles selfassemble to chains which form either snakelike structures [16], or "asters" [17], see Refs. [18-20] for reviews.

Magnetic snakes emerge only if certain preconditions are met: The frequency and amplitude of the field must be in the right range [21], and the viscosity of the liquid must be below

*Corresponding author: reinhard.richter@uni-bayreuth.de a certain value $[22,23]$. The properties of those snakes have been examined by observing their response to a dc magnetic field parallel to the water surface, revealing that the segments are antiferromagnetically aligned and consist of chains formed by ferromagnetically aligned particles [24]. Magnetic snakes are always accompanied by the hydrodynamic flow structure consisting of two pairs of vortices at the ends of the snake [25]. The vector field representing this flow structure has been reconstructed via particle image velocimetry as well as numerical simulations [25].

After "birth," magnetic snakes are laterally immobile due to the symmetry of the quadrupole-vortex streaming generated by both oscillating tails. Self-propelled magnetic surface swimmers, which are examined in our experiments, can be created by breaking this symmetry [26]. This can be achieved by a spontaneous transition to an asymmetric state under increase of the driving frequency or by attaching nonmagnetic objects like glass beads to one end of a magnetic snake [26]. We found it easier to experiment with nonmagnetic disks, like the ones shown in Fig. 1(b). In Fig. 1(c) we present a characteristic trajectory of a swimmer in a Petri dish. See also the related movie S1 [15].

The speed $v$ of those swimmers strongly depends on the control parameters frequency $f$ and the root-mean-square (RMS) amplitude $A$ of the driving ac magnetic field of the form

$$
B(t)=\sqrt{2} A \sin (2 \pi f t) .
$$

Snezhko et al. [26] suggested the scaling

$$
v(f, A)=b \frac{f A^{2}}{a_{0}(f)},
$$

where $a_{0}(f)$ denotes the typical length of a segment of the snake [26], while $b$ is a proportionality constant. So far only the quadratic dependence of the averaged swimming velocity from the driving amplitude $\left(v \propto A^{2}\right)$ has been confirmed in experiment [26] and by a simplified model [27].

This model is based on the Navier-Stokes equation for liquids in shallow water approximation coupled to the Newton equations for interacting magnetic particles. It can describe 


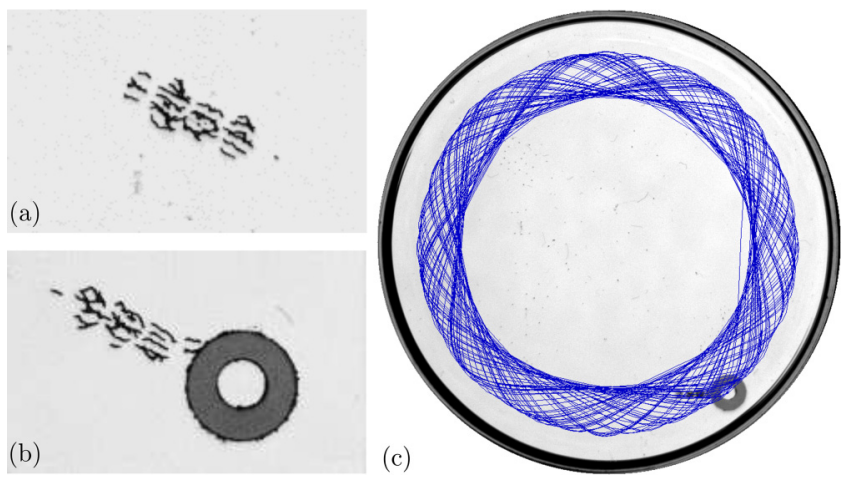

FIG. 1. Magnetic snake (a) and snake-disk-hybrid (b) created from that snake. Recorded at a frequency of $80 \mathrm{~Hz}$ and at a root-mean-square (RMS) amplitude of $8.5 \mathrm{mT}$. (c) Trajectory of the swimmer in a Petri dish recorded at a RMS amplitude of $12 \mathrm{mT}$. See also the related movie S1 [15].

the dynamic self-assembly of magnetic snakes. It is as well capable of simulating the speed of magnetic surface swimmers by replacing one of the magnetic particles by a larger, nonmagnetic bead with increased friction around itself. However, the authors state that "a quantitative comparison of experiment and theory is somewhat difficult because of the simplifications made" [27]. Still, a qualitative agreement for the scaling of the swimming velocity with the driving amplitude has been found. More recently, a model approach based on analytical solutions of the time-averaged Navier-Stokes equations has been put forward [23].

In this article we perform a thorough experimental test of Eq. (2). We measure the relations between swimming speed and amplitude for two swimmers of different length. More importantly, we explore for the first time the relation between swimming speed and frequency of the driving magnetic field. To allow long-term measurements, the course of the swimmer must be confined to a vessel of finite size. In order to prevent the swimmers being stopped by the edge, we utilize the ascending meniscus at the vertical wall of the Petri dish, which redirects the course of the swimmers. For different starting conditions and container geometries, we observe an interesting interplay between the moving swimmers and the elevated liquid surface, which results in periodic, quasiperiodic, and aperiodic trajectories.

\section{EXPERIMENTAL SETUP}

A sketch of the setup used for the experiments is shown in Fig. 2. The experimental vessel is filled to a depth of about $9 \mathrm{~mm}$ with distilled water and placed in the center of a Helmholtz-pair-of-coils. In the experiments, we use either a circular Petri dish made from glass (inner diameter: $70 \mathrm{~mm}$, height of the rim $11.5 \mathrm{~mm}$ ) or a stadium-shaped dish machined from Makrolon (Bayer AG) consisting of two semicircles of diameters $50 \mathrm{~mm}$ connected by straight walls with a length of $24.4 \mathrm{~mm}$. The coils are used to create a laterally homogeneous magnetic field perpendicular to the surface of the water. This is achieved with coils with a mean radius of $91 \mathrm{~mm}$ which are mounted in the Helmholtz distance [28]. Each coil is cooled by water. The current supplying the coils is created

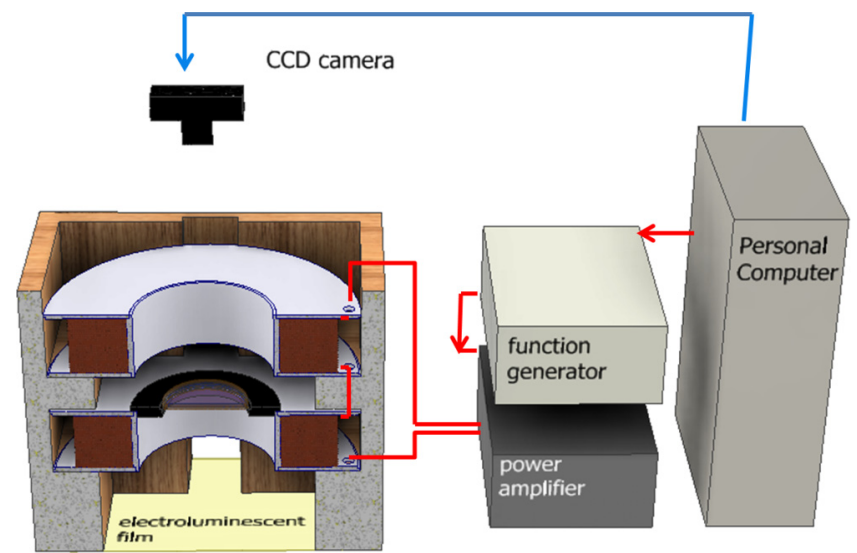

FIG. 2. Sketch of the setup used for the experiments. For details see text.

by a function generator (Fluke PM5138A) and amplified by a power amplifier (Spitzenberger+Spies EM400/CS). For all of our experiments, a current with sinusoidal time dependence was used, creating a magnetic induction according to Eq. (1) at the position of the water surface. The vessel is illuminated from below by an electroluminescent screen (Zigan displays) providing homogeneous illumination and observed by a charge-coupled device (CCD) camera (Lumenera Lu135MIO) mounted above the coils. To create a magnetic snake, spherical nickel particles with a mean diameter of $90 \mu \mathrm{m}$ are gently placed on the water surface in absence of the magnetic field, making sure that not too many particles sink to the ground. Afterwards, the amplitude of the magnetic field is slowly increased. At some threshold, a magnetic snake as seen in Fig. 1(a) assembles from the previously unorganized cluster of nickel particles. Due to the symmetry of the quadrupole vortex structure [25], this snake stays at a laterally fixed position. In order to create a moving magnetic surface swimmer, this symmetry is broken [26] by attaching a nonmagnetic object to one end of the snake. We utilize a washer formed from plastic with an outer diameter of $5.40 \mathrm{~mm}$, an inner diameter of $2.40 \mathrm{~mm}$, a height of $0.53 \mathrm{~mm}$, and a mass of $10.7 \mathrm{mg}$. Considering the "Cheerios effect" [29], this washer has to offer the same capillary charge as the nickel particles forming the snake, i.e., its density also has to be higher than the density of water, to be attracted to the magnetic snake. Otherwise, the snake and the washer would repel each other [29]. The washer has a density of about $1.10 \mathrm{~g} / \mathrm{cm}^{3}$ and can be placed on the water surface without sinking. Therefore it is appropriate for our experiments, as it easily attaches to the magnetic snake, as shown in Fig. 1(b). Likewise, the density of the washer prevents it from climbing up the ascending meniscus and thus introduces a convenient repulsion from the rim. The position $\vec{r}=(x, y)$ of the snake-disk-hybrid can be obtained via image-filtering provided by the open source library OpenCV [30].

\section{EXPERIMENTAL RESULTS AND DISCUSSION}

We first describe the different course of the swimmers in the circular Petri dish (Sec. III A) and thereby explain how we estimate their swimming velocity. Thereafter we present our 


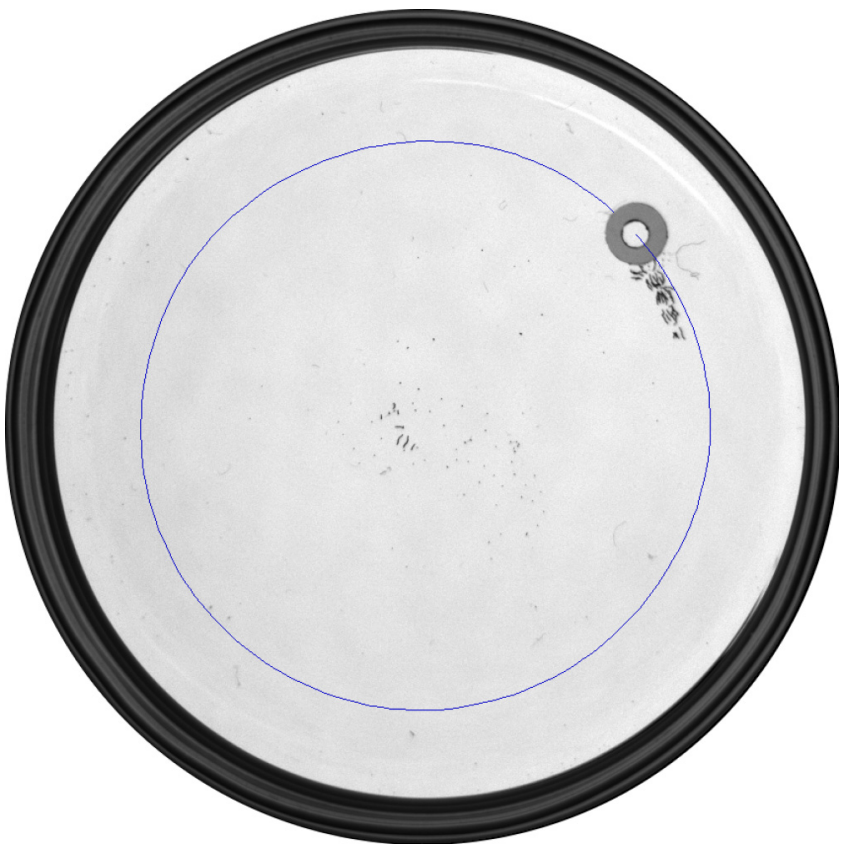

FIG. 3. Snake-disk-hybrid moving on a circular trajectory, recorded for one period at a frequency of $85 \mathrm{~Hz}$ and at a RMS amplitude of $10 \mathrm{mT}$. The line marks the trace of the center of the washer.

results for the swimming velocity as a function of the driving amplitude (Sec. III B) and the driving frequency (Sec. III C). Eventually, we compare the different types of trajectories in the circular vessel and in a stadium-shaped vessel with those of Hamiltonian billiards (III D).

\section{A. Circular and polygonal trajectories of the swimmers}

Having reconstructed the position of the surface swimmer as a function of time, its trajectory is the first thing one can investigate. In the laterally circular geometry of the Petri dish, two qualitatively different forms of trajectories occur.

In Fig. 3, we observe a periodic trajectory of the snakedisk-hybrid, which is swimming parallel to the edge of the circular container. Figure 4 presents the periodic sinusoidal evolution of the $x$ coordinate of the swimmer position. The dots mark the data, whereas the solid line represents a fit by $x_{\mathrm{fit}}(t)=x_{0} \sin (\omega t)$.

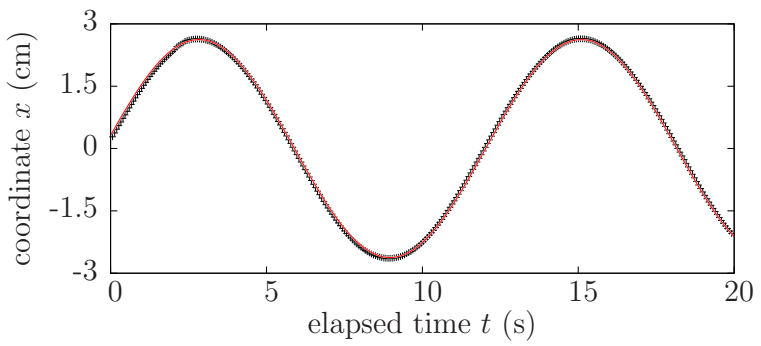

FIG. 4. The position along $x$ versus time for a magnetic swimmer moving on a circular trajectory, as presented in Fig. 3. The experimental data are marked by $(+)$. The solid line stems from a fit by $x_{\text {fit }}(t)=x_{0} \sin (\omega t)$.



FIG. 5. Snake-disk-hybrid moving on a quasiperiodic trajectory, recorded for $20 \mathrm{~s}$ at a frequency of $80 \mathrm{~Hz}$ and at a RMS amplitude of $12 \mathrm{mT}$. See also the related movie S1 [15].

In contrast to Fig. 3, a polygonal trajectory can be unveiled in Fig. 5, resembling a curve created by a spirograph. The trajectory is composed of straight and curved parts. If the swimmer is moving on the plain surface, then it is following a straight section; however, if it is approaching the edge of the container, then it is deflected, presumably by the resulting forces of the curved meniscus of the liquid surface. The nature of the trajectories depends on the angle and the speed with which the swimmer is approaching the wall of the container. The angle depends on the starting conditions, whereas the speed is determined by the control parameters of driving RMS amplitude $A$ and frequency $f$ but also by the size of the magnetic snake. The nature of the dynamics becomes as well prominent in the time series presented in Fig. 6, which clearly differs from a sine shown in Fig. 4. It displays in segments a linear evolution of $x(t)$ and distortions due to the reflections at the meniscus.

From the recorded trajectory, the speed can be calculated by multiplying the difference in position in consecutive frames with the frame sampling rate $f_{\mathrm{spl}}=15 \mathrm{fps}$. Figure 7 displays

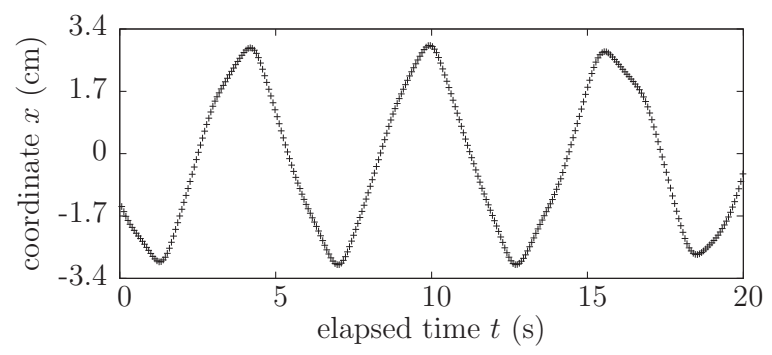

FIG. 6. The position along $x$ versus time for a magnetic swimmer moving on a polygonal trajectory, as shown in Fig. 5. 


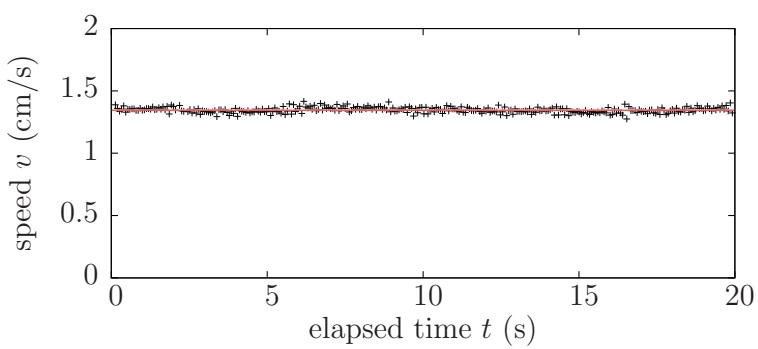

FIG. 7. The instantaneous speed $v$ of a magnetic swimmer moving on a circular trajectory, recorded at a RMS amplitude of $10 \mathrm{mT}$ and at a frequency of $85 \mathrm{~Hz}$. The horizontal red line indicates the average velocity $(1.345 \pm 0.002) \mathrm{cm} / \mathrm{s}$.

the instantaneous speed vs. time for the case of the circular trajectory, which is almost constant.

In contrast, the motion on the polygonal trajectories is associated with a change of the instantaneous speed, as shown in Fig. 8 (cf. dots connected by the red line). On the straight sections, the speed is maximal, whereas it is reduced when the swimmer is approaching the edge and subsequently deflected.

This is further illustrated by the density plot in Fig. 9, which shows the speed $v$ versus the radius $r$ for the data of Fig. 8 . It becomes obvious that the speed decreases with decreasing distance to the container edge at $r=3.5 \mathrm{~cm}$.

A possible explanation is that as the swimmer is approaching the edge of the container it has to ascend the meniscus and is therefore decelerated. This is corroborated by placing a simple, nonpropelled disk next to the container edge, which is floating away from the edge towards the center of the Petri dish. Of course, the situation is slightly more complex, because there are also hydrodynamic interactions between the swimmer and the vertical wall at the container edge.

Next we discuss the fact that, according to Fig. 5, a reflection of the swimmer takes place. The necessary force in the radial direction may be imposed by the gravity of the disk in connection with the meniscus. A repulsion may stem additionally from a hydrodynamic interaction between the wall and the driving vortex pair at the tail of the swimmer. Together with some flexibility in the swimmer-disk-hybrid, this may result in the curved trajectories, deflecting the swimmer away from the meniscus. Deceleration and acceleration after reflection is generating the sharp minima of $v(t)$ in Fig. 8.

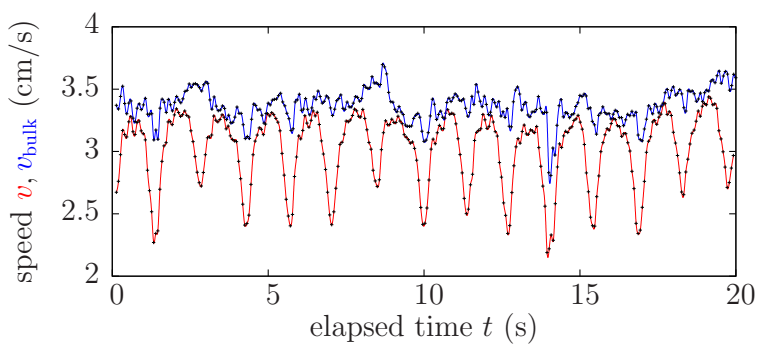

FIG. 8. The instantaneous speed $v$ of a magnetic swimmer moving on a quasiperiodic trajectory and recorded at a RMS amplitude of $12 \mathrm{mT}$ and at a frequency of $80 \mathrm{~Hz}$. The measured data points are connected by a red line. The points connected by a blue line mark the extrapolation of $v$ to $v_{\text {inf }}$ according to Eq. (4).

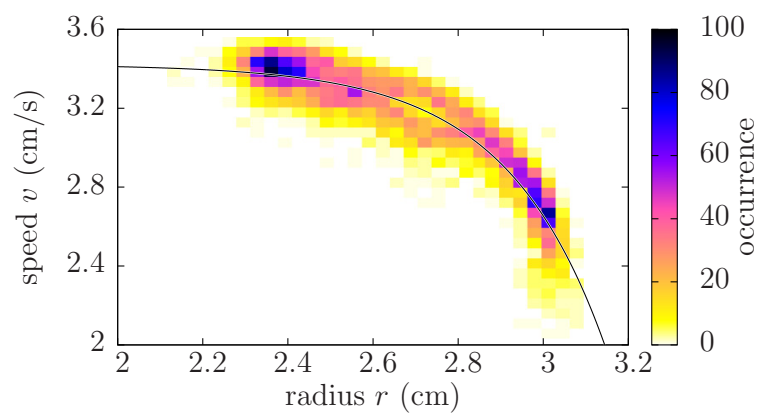

FIG. 9. Color-coded histogram of the measured radius versus the instantaneous speed for the trajectory of Fig. 5. The bin size is $0.0326 \mathrm{~cm} \times 0.0508 \mathrm{~cm} / \mathrm{s}$. The solid line marks a fit by Eq. (3).

Far from the edge, i.e., without a meniscus, the swimmer propagates on a straight course. The straight path is favoured by the symmetry of the problem, but its stability is nontrivial.

An interesting special case without reflection is the circular trajectory presented in Fig. 3. Here the centripetal force is provided by the interaction between the swimmer and the wall discussed above. It is remarkable that for a circular trajectory the velocity is determined by the same radial dependency $v(r)$ as for the polygonal one shown in Fig. 9.

In the subsequent two sections we will analyze the swimming speed as a function of the two control parameters, namely driving amplitude and frequency. Instead of extracting the swimming velocity only from the straight parts of the trajectory, which would reduce the amount of useful data considerably, we have chosen an alternative way: The data in Fig. 9 can well be fitted by a curve. Guided by the cylindrical symmetry of our vessel, we chose the phenomenological formula

$$
v_{\text {fit }}(r)=v_{\infty}-v_{\text {edge }} I_{0}(k r) .
$$

Here $I_{0}$ denotes the modified Bessel function of the first kind, and $v_{\infty}=3.42 \mathrm{~cm} / \mathrm{s}, v_{\text {edge }}=1.17 \times 10^{-5} \mathrm{~cm} / \mathrm{s}$, and $k=4.43 \mathrm{~cm}^{-1}$ are fit parameters for the specific curve in Fig. 9, which are kept fixed in the following. In order to utilize Eq. (3) for different data sets, it needs to be rescaled by $v_{\infty}$. For each velocity $v(r)$ an estimate for the velocity on an infinitely extended plain

$$
v_{\text {inf }}=\frac{v_{\infty}}{v_{\text {fit }}(r)} v(r)
$$

is extracted by means of Eq. (4). The result of such an extrapolation for an infinite container is marked in Fig. 8 by the blue curve. Its average for all $n$ data points in a record yields $\bar{v}=\frac{1}{n} \sum_{i=1}^{n} v_{\text {inf }, i}$.

\section{B. The swimming velocity for different driving amplitudes}

The magnetic snake needs a sufficiently large driving amplitude in order to maintain its structure. Therefore we begin both series of measurements at a large driving RMS amplitude of $A=12.5 \mathrm{mT}$ and lower $A$ until the swimmer is no longer stable and disassembles. We start the series by preparing a swimmer on a polygonal trajectory; however, during the measurements we do not have full control of the swimmers' character of motion. This is illustrated by Fig. 10 which 


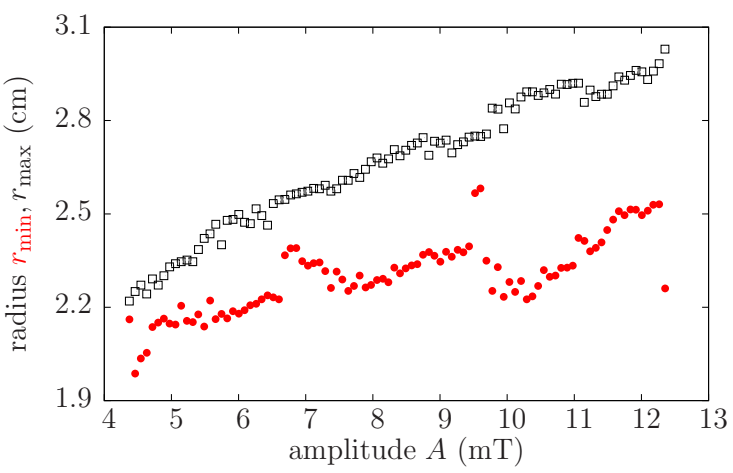

FIG. 10. The maximal $(\square)$ and minimal (•) radius in each data record versus the driving amplitude. The series of measurements stems from the swimmer presented in Fig. 11(b).

displays the maximal $(\square)$ and minimal (•) radius for about 90 different values of $A$. The maximal radius decreases with $A$ because it is determined by a balance of driving force and centripetal force. As a signature of a circular motion, one may expect $\left(r_{\max }-r_{\min }\right)=0 \mathrm{~cm}$. However, due to measurement noise, already a small difference is indicating a circular motion of the swimmer, as observed at $A=9.6 \mathrm{mT}$ and $A=6.8 \mathrm{mT}$ and around $A=4.4 \mathrm{mT}$. These transitions from polygonal to circular trajectories and back again may be induced by switching the driving field to the next lower value. For most of the amplitudes the swimmer moves on a polygonal trajectory. Having the swimmers type of motion clarified, we now focus on its velocity.

Figure 11 displays $\bar{v}$ for about 90 different values of the driving amplitude and for two different surface swimmers. All data were recorded at a constant frequency of $f=80 \mathrm{~Hz}$. The data points on curve (a) stem from the swimmer (a) displayed in Fig. 11, whose tail is composed of four segments. The datapoints on curve (b) originate from the corresponding surface swimmer (b) in Fig. 11, whose tail consists of six segments. Obviously, for the same amplitude, the swimmer with the longer tail swims faster than the shorter one, and both curves increase monotonously.

The functional dependence of the average speed in curve (a) can well be fitted by Eq. (2). The fit is as well successful

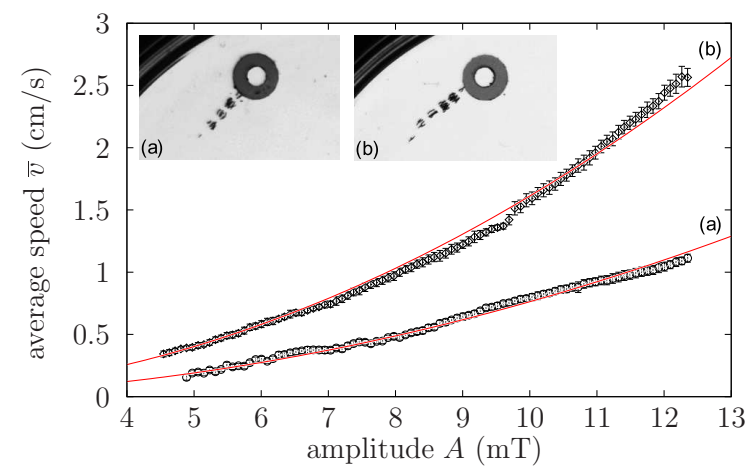

FIG. 11. Comparison of the curves obtained by plotting the average speed $\bar{v}$ versus the RMS amplitude $A$ for the two different magnetic swimmers shown as insets (a) and (b), recorded at a frequency of $80 \mathrm{~Hz}$. The solid lines are fits with Eq. (2).
TABLE I. Survey of the fit results for the short (a) and the long (b) swimmers. The parameter $c_{(x)}=\frac{b f}{a_{0}}$ is obtained by fitting the data in Fig. 11 with Eq. (2).

\begin{tabular}{lclc}
\hline \hline Snake & $N_{\text {segments }}$ & $c_{(x)}\left(\frac{\mathrm{m}}{\mathrm{T}^{2} \mathrm{~s}}\right)$ & \pm \\
\hline (a) & 4 & 076.3 & 0.4 \\
(b) & 6 & 161.2 & 0.4 \\
Ratio (a)/(b) & 0.666 & 000.473 & - \\
\hline \hline
\end{tabular}

for curve (b). The obtained fitting parameter are presented in Table I (third column), together with a rough estimate of the number of segments (second column). As already expected from Fig. 11, the larger swimmer leads to a larger fitting parameter $c_{(x)}=\frac{b f}{a_{0}}$, with $(x)$ denoting swimmer (a) or (b). This can be qualitatively understood by explaining how the quadrupole vortex structure is created. A magnetic snake is an oscillating object at the water surface, transporting kinetic energy to the fluid. In case (b), the oscillating area contributing to this process is larger, leading to a higher rate of energy transportation to the water, whereas the friction is dominated by the leading disk and thus almost constant. This consequently leads to a faster-moving surface swimmer [26]. In the last line of Table I, we list the ratio of the number of segments as well as the ratio $c_{(\mathrm{a})} / c_{(\mathrm{b})}$ of the fitting parameter for both snakes. The ratios are in the same order of magnitude but differ. This indicates that the assumption that $c \propto N_{\text {segments }}$ cannot be justified, most likely because the number of chains in the segments differs considerably.

\section{The swimming velocity for different driving frequencies}

The second control parameter is the frequency $f$ of the oscillating magnetic field. In our experimental setup, however, the frequency $f$ cannot be varied independently from the amplitude that easily, because the amplification provided by the power oscillator changes with frequency. To assure an amplitude of the magnetic induction as constant as possible, the voltage amplitude specified at the function generator also has to be adjusted. Due to the discrete voltage steps deviations in the range of $0.1 \mathrm{mT}$ of the magnetic induction amplitude occur for different data points.

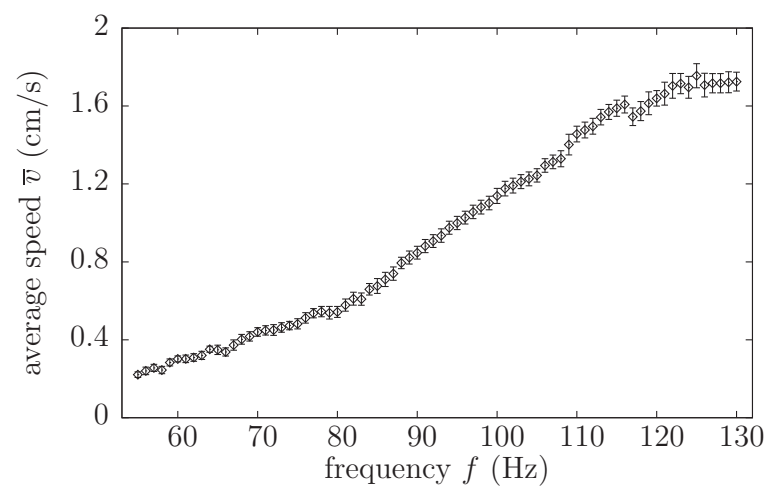

FIG. 12. The average speed $\bar{v}$ versus the frequency $f$, recorded at a RMS amplitude of about $8 \mathrm{mT}$. 
Aiming at a constant RMS amplitude of about $8 \mathrm{mT}$, Fig. 12 contains the raw data of a measurement series regarding the average speed $\bar{v}$ vs. the frequency $f$. Obviously, the data points in Fig. 12 do not lie on a line through origin, as one could expect when considering (2). The variations in the amplitude are not big enough to explain those deviations. But Eq. (2) also contains the typical size of a snake segment $a_{0}$. One period with wavelength $\lambda$ of the snakelike pattern always consists of two segments [26], yielding the relation $\lambda=2 a_{0}$. The wavelength $\lambda$ is strongly frequency dependent, which can be seen in Fig. 13. A frequency of $60 \mathrm{~Hz}$ as used in Fig. 13(a) obviously results in a larger wavelength than a frequency of $100 \mathrm{~Hz}$ as used in Fig. 13(b).

For plain water waves the theoretical dependency between $a_{0}$ and $f$ is determined by the dispersion relation

$$
\omega^{2}=g k+\frac{\sigma}{\rho} k^{3}
$$

where $\omega=2 \pi f$ denotes the angular frequency and $k=$ $2 \pi / \lambda=\pi / a_{0}$ the wave number. Equation (5) does not consider the depth of the liquid layer, because damping by the bottom of the container can be neglected as long as the depth of the layer is larger than the wavelength. We utilize this approximation for the evaluation of our experiment. Snezhko et al. find that the simple Eq. (5) describes the dispersion fairly well (cf. Fig. 3 in Ref. [21]). They suggest a small modification of the surface tension in (5) due to the presence of the particles on the surface. The small deviations are corroborated by recent measurements [28], which suggests, however, that small corrections by a magnetic term are necessary. This is in agreement with a model put forward in Ref. [23].

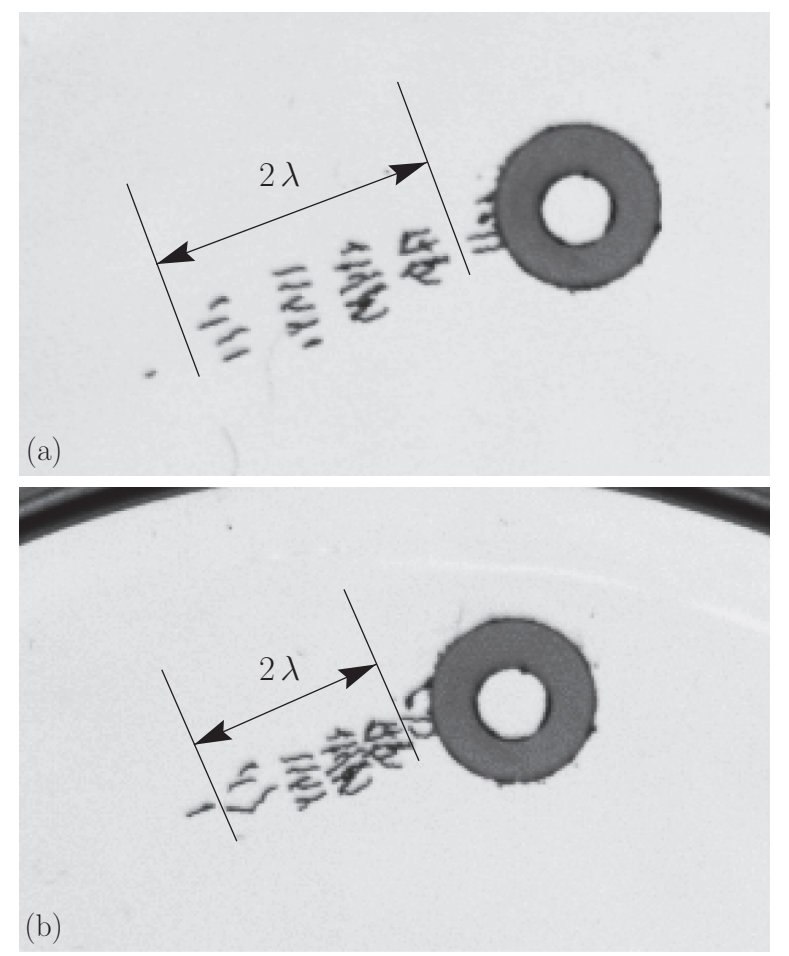

FIG. 13. Snake-disk-hybrid at different frequencies, i.e., $60 \mathrm{~Hz}$ (a) and $100 \mathrm{~Hz}$ (b).

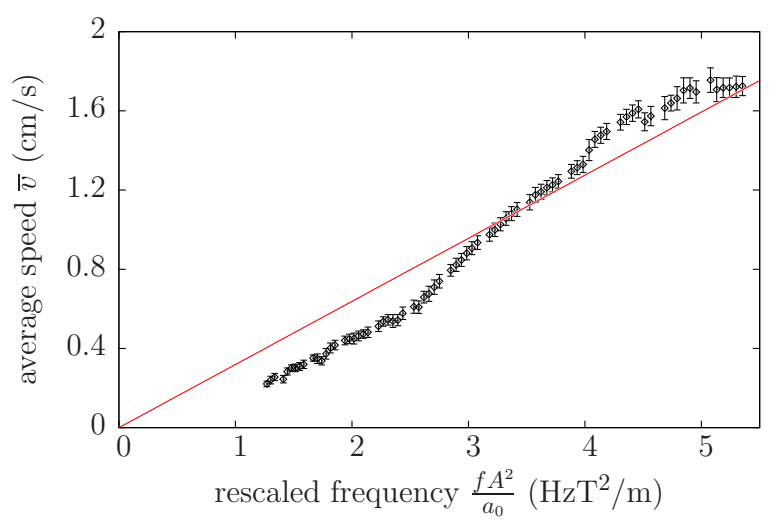

FIG. 14. The average speed $\bar{v}$ versus the rescaled frequency $\frac{f A^{2}}{a_{0}}$. The solid line displays a fit by Eq. (2) with the parameter $b=(31.9 \pm$ $0.5) \times 10^{-4}(\mathrm{~m} / \mathrm{T})^{2}$.

In a first approach, we utilize Eq. (5) to take the frequency dependence of the size $a_{0}$ of snake segments into account: Substituting the wave number $k$ by $\pi / a_{0}$, and the angular frequency $\omega$ by $2 \pi f$, and inserting the values $g=9.81 \frac{\mathrm{m}}{\mathrm{s}^{2}}$, $\sigma=72.75 \frac{\mathrm{mN}}{\mathrm{m}}, \rho=1003 \frac{\mathrm{kg}}{\mathrm{m}^{3}}$ for the gravitational acceleration and the surface tension and density of water, respectively, yields the function $f\left(a_{0}\right)$. By inverting this function, the raw data shown in Fig. 12 can be modified by rescaling the $x$ axis from $f$ to $\frac{f A^{2}}{a_{0}}$. With this term we can also take into account that the amplitude varies slightly for different data points. The result of this procedure is visualized in Fig. 14. The data points now indeed lie close to a line through the origin. However, at small frequencies $\bar{v}$ is smaller, and at large frequencies $\bar{v}$ appears to saturate. Fitting the data yields a value of $b=(31.9 \pm 0.5) \times 10^{-4} \frac{\mathrm{m}^{2}}{\mathrm{~T}^{2}}$.

\section{Swimming in a pool billiard}

In Sec. III A we have shown two qualitatively different types of swimmer trajectories in the circular Petri dish. We found circular trajectories parallel to the meniscus (cf. Fig. 3) and polygonal trajectories as presented in Fig. 5. On the latter the swimmer is reciprocating on straight paths between the container walls, where it becomes deflected by the meniscus. We have seen that those trajectories are not closed.

In order to shed more light on the dynamics, we plot in Fig. 15 the position of the swimmer in cylinder coordinates $(r, \varphi)$, where $r$ measures the distance of the swimmer from the center of the dish and $\varphi$ gives the angle with the vertical. Figure 15(a) displays $r(t)$, which shows a fairly regular oscillation to and from the wall of the vessel. This is corroborated by a fit with

$$
r_{\text {fit }}(t)=R_{0} \sin \left(\omega_{r} t\right)
$$

[cf. the solid red line in Fig. 15(a)]. Minor irregularities may stem from long-range interactions of the driving vortex pair with the container wall. Note in the movie S1 [15] the motion of single floating Ni particles as the swimmer passes. In Fig. 15(b) we plot the data $\varphi(t)\left(\bmod 360^{\circ}\right)$ together with a fit by

$$
\varphi_{\mathrm{fit}}(t)=\omega_{\varphi} t \quad\left(\bmod 360^{\circ}\right),
$$




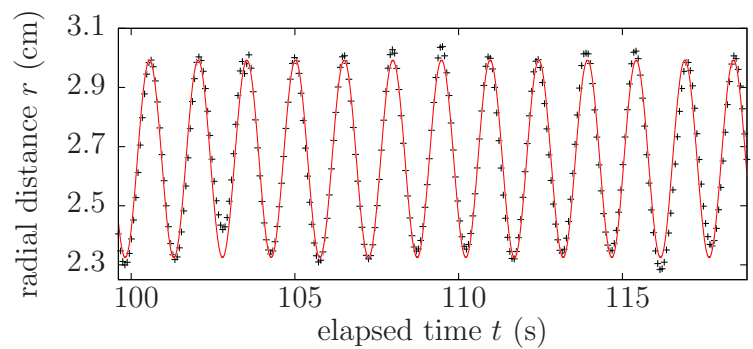

(a)

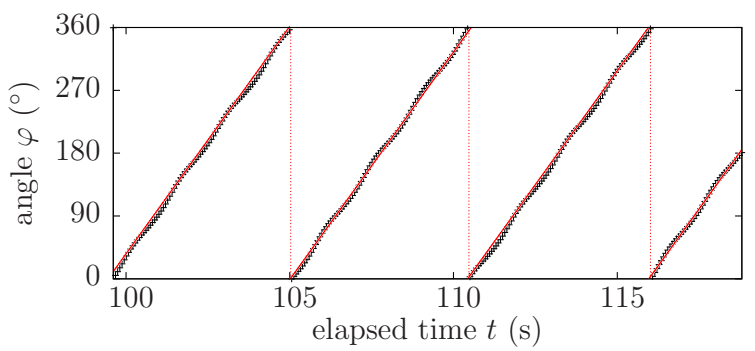

(b)

FIG. 15. Decomposition of the swimmer motion in radius (a) and angle (b) for a magnetic swimmer moving on a polygonal trajectory, as shown in Fig. 5. The solid red line in chart (a) displays a fit by Eq. (6) with $R_{0}=(0.33 \pm 0.02) \mathrm{cm}$ and $\omega_{r}=(4.228 \pm 0.002) \mathrm{s}^{-1}$. The solid red line in chart (b) displays a fit by Eq. (7) with $\omega_{\varphi}=$ $(1.138 \pm 0.005) \mathrm{s}^{-1}$.

which proves an almost linear increase of the angle with time. Small modulations around the linear increase have the frequency $\omega_{r}$.

Figure 16 displays the Fourier decomposition of $r(t)$. One clearly discriminates a peak at $f_{r}=\omega_{r} /(2 \pi)=0.672 \mathrm{~Hz}$, marked by a vertical dashed red line, where $\omega_{r}$ was determined from the fit of $r(t)$ by Eq. (6). The vertical dashed red line at $f_{\varphi}=\omega_{\varphi} /(2 \pi)=0.181 \mathrm{~Hz}$ indicates a second frequency impressed by the revolution of the swimmer around the dish, as captured by Eq. (7). For the presented data set the winding number $\Omega=f_{r} / f_{\varphi} \approx 0.2686 \ldots$ is considered as irrational, i.e., we have a quasiperiodic dynamic and the trajectories never close. Note that $\Omega$ is close to $1 / 4$, which becomes prominent in the trajectory in Fig. 5: The trajectory displays slightly fewer than four reflections at the meniscus for one full round of the swimmer.

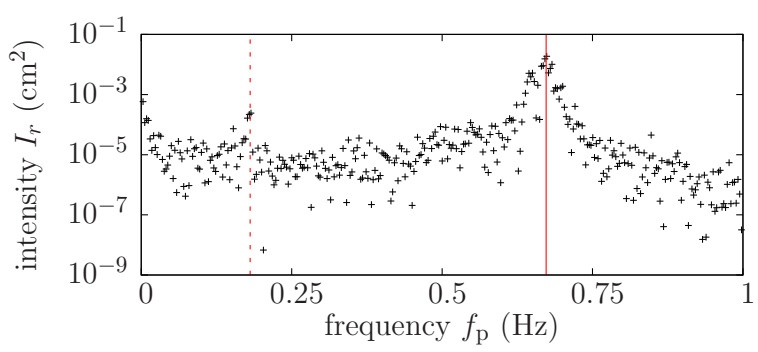

FIG. 16. The Fourier decomposition estimated from $r(t)$ from the time series presented partly in Fig. 15(a). The vertical solid line at $f_{r}=0.672 \mathrm{~Hz}$ indicates the frequency determined from the fit of $r(t)$ by Eq. (6). The vertical dashed red line at $f_{\varphi}=0.181 \mathrm{~Hz}$ stems from the fit by Eq. (7).

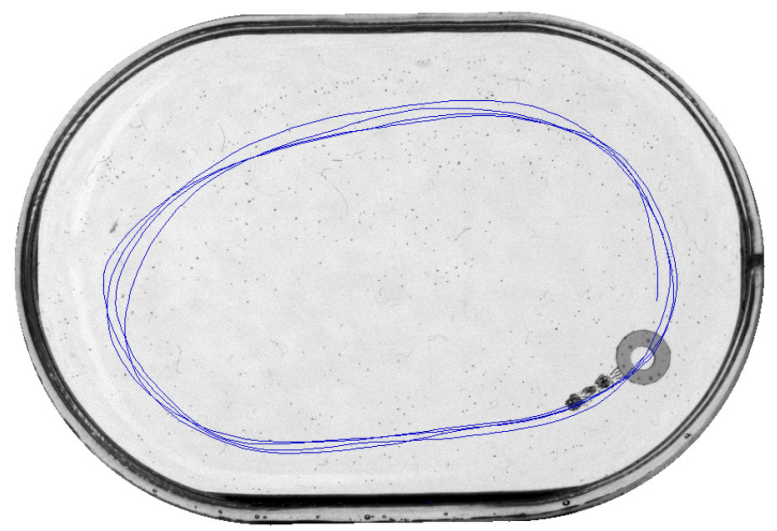

FIG. 17. Snake-disk-hybrid moving on a periodic trajectory, recorded for $1 \mathrm{~min}$ at a frequency of $80 \mathrm{~Hz}$ and at a RMS amplitude of $12 \mathrm{mT}$. See also the related movie S2 [15].

Next we replace the Petri dish with a stadium-shaped vessel as shown in Fig. 17. Like in Fig. 3, the snake-disk-hybrid swims for certain starting conditions along the container wall, and we observe a periodic trajectory. For different starting values the surface swimmer crosses the stadium and becomes reflected at the meniscus next to the container wall. Figure 18 presents the trajectories recorded for $300 \mathrm{~s}$.

The trajectories criss-cross the whole vessel and are clearly aperiodic. Even the sense of rotation is changed after $t=60 \mathrm{~s}$ from counterclockwise to clockwise, as can be unveiled in the related movie S3 [15]. The aperiodic dynamic becomes also clear from the time series of the $x$ position of the surface swimmer, as presented in Fig. 19. The Fourier decomposition estimated from $x(t)$ for a time series of $300 \mathrm{~s}$ is shown in Fig. 20 (red diamonds). It displays low-frequency noise characteristics for deterministic chaotic dynamics. For comparison, the Fourier decomposition obtained from $x(t)$ of the quasiperiodic dynamics (cf. black crosses) is considerably lower at small frequencies. Around $0.5 \mathrm{~Hz}$ and $0.8 \mathrm{~Hz}$, it displays the odd higher harmonics of the fundamental mode, which are absent in the chaotic spectrum.

The different trajectories shown above resemble those observed in two-dimensional dynamical billiards [31]. According to their long-time behavior, they can be divided into

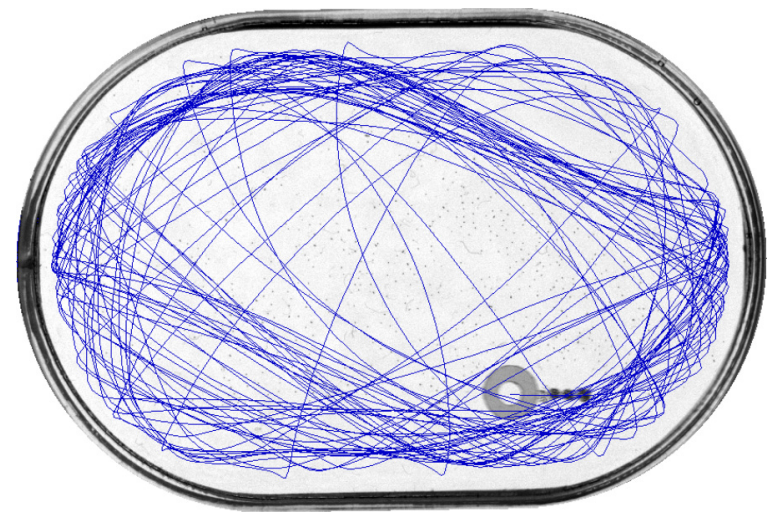

FIG. 18. Snake-disk-hybrid moving on an aperiodic trajectory, recorded for $5 \mathrm{~min}$ at a frequency of $80 \mathrm{~Hz}$ and at a RMS amplitude of $12 \mathrm{mT}$. See also the related movie S3 [15]. 


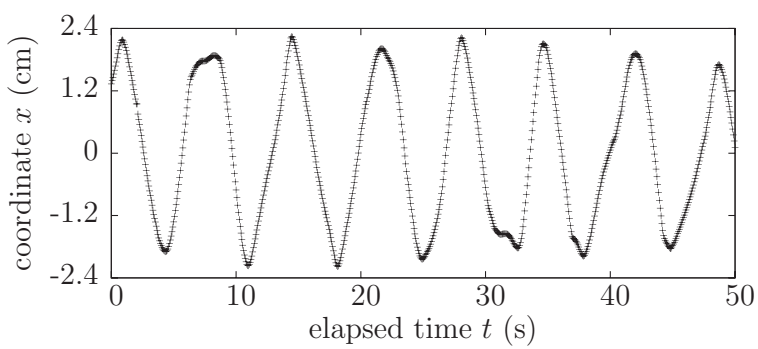

FIG. 19. The position along $x$ versus time for a magnetic swimmer moving on an aperiodic trajectory, as presented in Fig. 18.

two categories [31-33]: (i) Inside regular billiards like circles or squares, the trajectories form periodic or quasiperiodic orbits. Whether a periodic or quasiperiodic orbits is realized depends on the initial conditions. (ii) Inside chaotic billiards like the Bunimovich stadium billiard [34] the trajectories can be classified into a discrete set of periodic orbits of measure zero separated by an innumerable number of chaotic orbits.

Our swimming pool differs in three fundamental ways from a Hamiltonian billiard: First, it is a dissipative system, which needs a constant energy throughput to sustain the swimmers motion. This can be achieved by the oscillating magnetic field which is oriented normal to the liquid layer. This facilitates the swimmer to swim in any direction of the plain without a modification of the energy supply. Second, instead of fully elastic reflections of an ideal billiard sphere at one point of the container wall, we observe a curved trajectory mediated by the meniscus. Third, the swimmer is not a pointlike object but has a finite extension and may show long-range interactions with the side walls of the vessel by means of its driving vortex pair. Despite that, the similarities to Hamiltonian billiards are striking. In this picture the circular orbit in Fig. 3 resembles a whispering gallery orbit.

There are other recent examples for billiard-like trajectories of artificial swimmers, like the motion in a colloid filled bath with intermittent periods of entrapped and orbiting states $[35,36]$. In this way, arrays of obstacles have been suggested and utilized for cell sorting; see Ref. [37] for a recent review. In particular, the cell Chlamydomonas reinhardtii has been shown to be "reflected" from a wall: As the cell approaches a wall, flagellar interactions with the surface lead to cell rotation until the cell swims back into the bulk at a geometry-specific departure angle [38]. However, in all these examples the microscopic swimmers are moving in the bulk

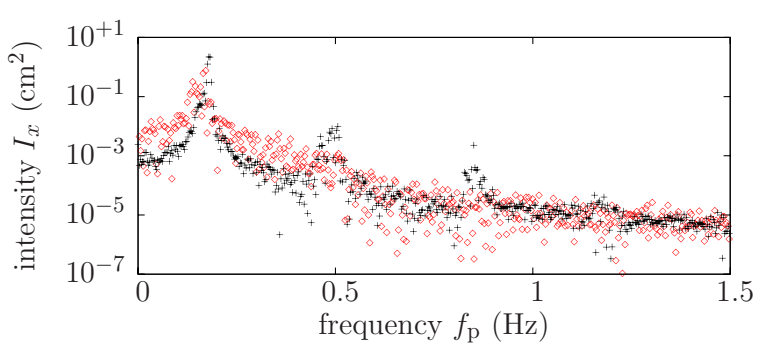

FIG. 20. The Fourier decomposition (red diamonds) estimated from the time series $x(t)$ partly presented in Fig. 19. For comparison, we display as well (black crosses) the spectrum estimated from $x(t)$ of the quasiperiodic dynamics presented in Fig. 6. of the liquid and are not restricted to its surface. Therefore the meniscus does not have a decisive influence on their trajectories. In contrast, our macroscopic surface swimmers are guided by the meniscus on deterministic trajectories away from the wall.

\section{SUMMARY AND CONCLUSION}

First, we measured the averaged swimming velocity in a circular container for different driving amplitudes. We reconfirmed a scaling of the velocity with the square of the magnetic driving amplitude, measured previously [26]. This scaling has been observed in experiments in many magnetic systems $[13,39]$ and originates from the fact that the dynamics is influenced by the direction but not by the orientation of the magnetic field. We corroborated that the speed of the swimmer-disk-hybrids increases monotonically with the length of the tail.

Second, the regular swimming course in a confined geometry allowed us to measure the swimming velocity for different driving frequencies. Taking into account the frequency-dependent size of a swimmer segment by means of the simple dispersion relation of water waves, we found an increase of the averaged swimming velocity which is cum grano salis proportional to the rescaled driving frequency, as stated in Eq. (2).

With more elaborate model equations at hand [23,27], it remains to be investigated whether the models show a deviation from Eq. (2) as well and a saturation at higher driving frequencies.

Third, we studied the course of self-assembled magnetic surface swimmers on a layer of water in vessels of different geometry. For a vessel with circular shape, periodic trajectories along the container wall have been observed, as well as quasiperiodic trajectories with straight parts traversing the vessel. They are connected by curved segments next to the container wall. The latter originate from a deflection of the swimmer by the meniscus. Selecting a vessel with an outline similar to a stadium oval, a periodic trajectory along the circumference has been found. More importantly, trajectories which are crossing the stadium-shaped container show aperiodic dynamics. In this way, we find striking similarities to Hamiltonian dynamical billiards. However, our system differs in three aspects: (i) The swimmer needs a constant supply of energy. (ii) Instead of being reflected at a singular point of the wall, the course of the swimmer is bent by the meniscus next to the vertical container wall. (iii) The swimmer is an extended object. Despite these three differences, it is remarkable that, so far, our "swimming pool billiard" shows the qualitative features of a dynamical billiard in a circular and a Bunimovich geometry. That statement might not hold for other geometries.

In our system the reflections are induced by the curvature of the meniscus at the container wall. A curved liquid topography might alternatively be created by heating locally via illumination, which would lower the surface tension of the liquid, leading to Marangoni convection and a curved surface as a consequence. In this way, reflections at the liquid boundaries could be studied under continuous variation of the curvature, and the flexibility for testing arbitrary billiard shapes would be enhanced. 


\section{ACKNOWLEDGMENTS}

The authors thank A. Snezhko for providing us with sieved Ni particles and for a hands-on introduction into the experiment and Klaus Oetter for assistance in constructing the experimental setup. Moreover, discussions with Agnes Baumgärtner, Christof Kruelle, and Tobias Lang are gratefully acknowledged.
[1] E. Lauga and T. R. Powers, The hydrodynamics of swimming microorganisms, Rep. Prog. Phys. 72, 096601 (2009).

[2] J. Elgeti, R. G. Winkler, and G. Gompper, Physics of microswimmers-single particle motion and collective behavior: A review, Rep. Prog. Phys. 78, 056601 (2015).

[3] D. L. Koch and G. Subramanian, Collective hydrodynamics of swimming microorganisms: Living fluids, Annu. Rev. Fluid Mech. 43, 637 (2011).

[4] A. Zöttl and H. Stark, Emergent behavior in active colloids, J. Phys.: Condons. Matter 28, 253001 (2016).

[5] U. Häfeli, W. Schütt, J. Teller, and M. Zborowski, Scientific and Clinical Applications of Magnetic Darriers (Springer Science \& Business Media, Berlin, 2013).

[6] J. Dietrich, P. Meier, S. Oberthur, R. Preub, D. Voges, and K. Zimmermann, Development of a peristaltically actuated device for the minimal invasive surgery with a haptic sensor array, In Micro-and Nanostructuers of Biological Svstems (ShakerVerlag, Aachen, 2004), pp. 69-88.

[7] N. Bayat and A. Nethe, Technical applications. In Colloidal Magnetic Fluids: Basics, Development and Applications of Ferrofluids, Vol. 763, edited by S. Odenbach (Springer, Berlin, New York, 2009), chapter 6, p. 331.

[8] N. Pamme, Magnetism and microfluidics, Lab Chip 6, 24 (2006).

[9] E. M. Purcell, Live at low Reynolds numbers, Am. J. Phys. 45, 3 (1977).

[10] T. Qiu, T.-C. Lee, A. G. Mark, K. I. Morozov, R. Münster, O. Mierka, S. Turek, M. Alexander, A. M. Leshansky, and P. Fischer, Swimming by reciprocal motion at low Reynolds number, Nat. Commun. 5, 5119 (2014).

[11] R. Dreyfus, J. Baudry, L. Marcus, M. L. Roper, M. Fermigier, H. A. Stone, and J. Bibette, Microscopic artificial swimmers, Nature 437, 862 (2005).

[12] A. Ghosh and P. Fischer, Controlled propulsion of artificial magnetic nanostructured propellers, Nano Lett. 9, 2243 (2009).

[13] V. Sterr, R. Krauß, K. I. Morozov, I. Rehberg, A. Engel, and R. Richter, Rolling ferrofluid drop on the surface of a liquid, New J. Phys. 10, 063029 (2008).

[14] P. Tierno, R. Golestanian, I. Pagonabarraga, and F. Sagués, Controlled Swimming in Confined Fluids of Magnetically Actuated Colloidal Rotors, Phys. Rev. Lett. 101, 218304 (2008).

[15] See Supplemental Material at http://link.aps.org/supplemental/ 10.1103/PhysRevE.96.012205 for movies S1-S3. The supplemental movie $\mathrm{S} 1$ displays a swimmer moving on a quasiperiodic trajectory in a circular Petri dish. The movie S2 displays a regular trajectory of a swimmer in a dish with stadion shape, whereas the movie S3 displays a swimmer progressing on an aperiodic trajectory in the latter dish.

[16] A. Snezhko, I. S. Aranson, and W.-K. Kwok, Structure Formation in Electromagnetically Driven Granular Media, Phys. Rev. Lett. 94, 108002 (2005).

[17] A. Snezhko and I. S. Aranson, Magnetic manipulation of selfassembled colloidal asters, Nat. Mater. 10, 698 (2011).
[18] J. E. Martin and A. Snezhko, Driving self-assembly and emergent dynamics in colloidal suspensions by time-dependent magnetic fields, Rep. Prog. Phys. 76, 126601 (2013).

[19] I. S. Aranson, Collective behavior in out-of-equilibrium colloidal suspensions, C. R. Phys. 14, 518 (2013).

[20] A. Snezhko, Complex collective dynamics of active torquedriven colloids at interfaces, Curr. Opin. Colloid Interf. Sci. 21, 65 (2016).

[21] A. Snezhko, I. S. Aranson, and W.-K. Kwok, Dynamic selfassembly of magnetic particles on the fluid interface: Surfacewave-mediated effective magnetic exchange, Phys. Rev. E 73, 041306 (2006).

[22] D. L. Piet, A. V. Straube, A. Snezhko, and I. S. Aranson, Viscosity Control of the Dynamic Self-Assembly in Ferromagnetic Suspensions, Phys. Rev. Lett. 110, 198001 (2013).

[23] D. L. Piet, A. V. Straube, A. Snezhko, and I. S. Aranson, Model of dynamic self-assembly in ferromagnetic suspensions at liquid interfaces, Phys. Rev. E 88, 033024 (2013).

[24] A. Snezhko, I. S. Aranson, and W.-K. Kwok, Surface Wave Assisted Self-Assembly of Multidomain Magnetic Structures, Phys. Rev. Lett. 96, 078701 (2006).

[25] M. Belkin, A. Snezhko, I. S. Aranson, and W.-K. Kwok, Driven Magnetic Particles on a Fluid Surface: Pattern Assisted Surface Flows, Phys. Rev. Lett. 99, 158301 (2007).

[26] A. Snezhko, M. Belkin, I. S. Aranson, and W.-K. Kwok, SelfAssembled Magnetic Surface Swimmers, Phys. Rev. Lett. 102, 118103 (2009).

[27] M. Belkin, A. Glatz, A. Snezhko, and I. S. Aranson, Model for dynamic self-assembled magnetic surface structures, Phys. Rev. E 82, 015301 (2010).

[28] A. Baumgärtner, Experimentelle Untersuchung von selbstorganisierten magnetischen Partikeln auf der Wasseroberfläche. Diplomarbeit, Universität Bayreuth, Bayreuth (2011).

[29] D. Vella and L. Mahadevan, The cheorios effect, Am. J. Phys. 73, 817 (2005).

[30] Open Computer Vision (openCV) [https://github.com/opencv /opencv/wiki]

[31] M. C. Gutzwiller, Chaos in Classical and Quantum Mechanics, Vol. 1 (Springer Science \& Business Media, Berlin, 2013).

[32] N. Chernov and R. Markarian, Chaotic Billiards, Volume 127 of Mathematical Surveys and Monographs (American Mathematical Society, Providence, RI, 2006).

[33] C. A. Kruelle, A. Kittel, J. Peinke, R. Richter, and R. P. Huebener, Chaotic billiards seen as mirror cabinets, Physica D 102, 227 (1997).

[34] L. A. Bunimovich, S. G. Dani, R. L. Dobrushin, M. V. Jakobson, I. P. Kornfeld, N. B. Maslova, Y. B. Pesin, J. Smillie, Yu. M. Sukhov, and A. M. Vershik, Dynamical Systems, Ergodic Theory and Applications, Vol. 100 (Springer Science \& Business Media, Berlin, 2000).

[35] D. Takagi, A. B. Braunschweig, J. Zhang, and M. J. Shelley, Dispersion of Self-Propelled Rods Undergoing FluctuationDriven Flips, Phys. Rev. Lett. 110, 038301 (2013). 
[36] S. E. Spagnolie, R. Gregorio, G. R. Moreno-Flores, D. Bartolo, and E. Lauga, Geometric capture and escape of a microswimmer colliding with an obstacle, Soft Matter 11, 3396 (2015).

[37] C. Bechinger, R. Di Leonardo, H. Löwen, C. Reichhardt, G. Volpe, and G. Volpe, Active particles in complex and crowded environments, Rev. Mod. Phys. 88, 045006 (2016).
[38] V. Kantsler, J. Dunkel, M. Polin, and R. E. Goldstein, Ciliary contact interactions dominate surface scattering of swimming eukaryotes, Proc. Natl. Acad. Sci. USA 110, 1187 (2013).

[39] R. Krauß, M. Liu, B. Reimann, R. Richter, and I. Rehberg, Pumping fluid by magnetic surface stress, New J. Phys. 8, 18 (2006). 